person specific and influenced by many factors (e.g. psychosocial factors, illness perceptions, sleep, comorbidity etc.). A thorough history alone usually suggests the single most likely cause for the patient's problem(s). The history should then guide the subsequent physical examination - an efficient targeted "rapier" approach is recommended in which the practitioner selects the appropriate skills from a range of competencies according to specific elements in the history. This contrasts with a more lengthy hypothesis-free "general screen" in which the same set of uniform procedures is undertaken in each patient.

This presentation will cover key principles and considerations of assessment and illustrate how the history guides the subsequent "rapier" examination (2). Examples include:

(1) in the history: determination of pain localisation and features that associate with radiated pain; important pain and stiffness characteristics that differentiate mechanical usage-related pain, inflammatory pain, acute crystal synovitis pain, destructive bone pain and neurogenic pain; non-specific symptoms of inflammation (2) in the examination: usual order of inspection at rest, inspection during movement, then palpation at rest and during movement of symptomatic regions; contrasting clinical findings that quickly differentiate joint and peri-articular problems; initial selection of the movement(s) that is affected first and most severely by arthropathy - the tight pack position(s); detection of "stress pain" (pain worse in tight-pack positions but reduced/absent in loose-pack positions the most sensitive sign of inflammation); examination for effusion, soft-tissue and firm swelling; use of resisted active movements and stress tests for peri-articular lesions; a targeted screen for asymptomatic disease prompted by main diagnosis. EULAR learning resources available at http://www.eular.org/edu_training_dvd.cfm include: (1) The "GALS" screen and (2) Principles of the musculoskeletal history and examination.

Disclosure of Interest: None declared

DOI: 10.1136/annrheumdis-2017-eular.2947

\section{SP0103 HOW TO ASSESS US COMPETENCY SKILLS}

\section{H.B. Hammer. Dept of Rheumatolgy, Diakonhjemmet Hospital, OSLO, Norway}

To optimize and standardize musculoskeletal ultrasonography education for rheumatologists, there is a need for competency assessments addressing the required training and practical and theoretical skills. Because of the increasing use of MSUS in rheumatology, there has been a focus over the past years on training.

A minimum training requirements are described by The European Federation of Societies for Ultrasound in Medicine and Biology (EFSUMB) where a 3 levels competency assessment (COMPASS) has been developed for rheumatologists, including an in-detail description of what theoretical and practical competencies to be acquired at each level with a related log book (1). The rheumatology-COMPASS levels are closely related to the levels of the EULAR MSUS courses, thereby ensuring that the content is supported by already provided courses such as the EULAR and EULAR-endorsed MSUS courses to facilitate the implementation of the rheumatology-COMPASS. In COMPASS level 1 the course contents resemble the EULAR MSUS basic and intermediate courses, level 2 resembles the EULAR MSUS advanced course whereas level 3 requires attendance in a "teach-theteachers course" or experience as a teacher in at least 2 international MSUS courses. Level 3 also includes an academic level requiring research activity and acceptance of level 1 and 2 sonographers for training.

The EULAR MSUS courses have been organized since 1998 and the interest in these courses has been increasing. In 2007, the first 3 level EULAR MSUS course was conducted with great success and the 3 level courses (basic, intermediate and advanced) have been running ever since in relation to the EULAR congress, focusing mainly on the relevant content on the individual levels and the distribution between practical and theoretical skills.

EULAR has developed the following competence levels; level 1 and 2. The EULAR level 1 competency includes the performance of EULAR Online MSUS course and attendance to basic, intermediate and advanced MSUS courses, where attending the intermediate and advanced courses require a certain number of US examinations (however, if already reached COMPASS level 1, 2 or 3, there is no need of images for the EULAR courses), and the advanced course requires in addition to pass a practical examination. The EULAR level 2 competency is organized to ensure a minimum level of US knowledge for teachers in MSUS courses (2). This level includes the EULAR Teach the Teachers course as well as passing a theoretical and practical examination. Since there is a growing number of EULAR endorsed MSUS courses, it is of highly importance that the teachers in these courses have equal qualifications thereby providing comparable training and competencies beneficial for the clinical use of US. Information about the competence requirements is found at the EULAR website (http://www.EULAR.org).

Disclosure of Interest: None declared

DOI: 10.1136/annrheumdis-2017-eular.7147

\section{THURSDAY, 15 JUNE 2017 Macrophage M2 polarization: implications in fibrotizing diseases}

\section{SP0104 REPROGRAMMING OF MYELOID CELLS IN CANCER: MECHANISMS AND SIGNIFICANCE}

\section{A. Sica. Pharmacological Sciences, University of Eastern Piedmont, NOvara,} Italy

Chronic inflammation mediates tumor development by promoting a constant influx of inflammatory cells capable of modulating genes involved in cancerogenesis and creating micro and macroenvironments that support cancer growth. A major side effects of cancer inflammation is the pathological expansion and recruitment of myeloid cells endowed with immunosuppressive activity, to control the unresolved inflammation. Tumors reprogram myeloid cell differentiation and functions through various mechanisms, including altered metabolism, cancerrelated inflammation andalteration of the hematopoietic output. These events govern the expansion of myeloid suppressor populations, mainly myeloid-derived suppressor cells (MDSCs) and tumor associated macrophages (TAMs). MDSCs and TAMs orchestrate tumor immunosuppression in concert with regulatory $T$ cells, inhibitory cytokines and immune check points receptors, and act to subvert anti-tumor immunity, hence causing that eventually support immune evasion establishing a bottleneck for cancer immunotherapy. I will discuss inflammatory circuits and epigenetic events sustaining the expansion and the tumor-promoting reprogramming of myeloid cells in cancer bearers.

Disclosure of Interest: None declared

DOI: 10.1136/annrheumdis-2017-eular.7285

\section{SP0105 MACROPHAGES, METABOLISM AND INFLAMMATION}

N.O.S. Camara. Immunology, University of Sao Paulo, Sao Paulo, Brazil

Macrophages are a heterogeneity population implicated in several diseases and correlated with distinct tissue outcomes after injury. M2 macrophages have been associated with tissue repair whereas $M 1$ macrophages participate in early phase of tissue damage. Recent works have also suggested that upon activation, macrophages can use distinguished nutrients as source of energy and these metabolic pathways lead to their activation and differentiation. Nutrient sensors are intimae associated with innate receptors and therefore connected with inflammatory response. Uric acid is a damage-associated molecular pattern (DAMP), released from ischemic tissues and dying cells which, when crystalized, is able to activate the NLRP3 inflammasome through frustrated phagocytosis. Soluble uric acid (sUA) is found in high concentrations in the serum of great apes, and even higher levels in some diseases, before the appearance of crystals. sUA can be released in a hypoxic environment and triggers NLRP3 through the production of mitochondrial ROS, with increased maximum and reserve oxygen consumption ratio (OCR) and higher VDAC protein levels. This process is followed by ASC speck formation, caspase- 1 activation and IL-1 $\beta$ release. These findings may have profound implications for inflammatory-related diseases. Support: FAPESP and CNPq.

Disclosure of Interest: N. Camara Grant/research support from: FAPESP, CNPq DOI: 10.1136/annrheumdis-2017-eular.7283

\section{FRIDAY, 16 JUNE 2017 \\ Mucosal B cells: gatekeepers of immune function}

\section{SP0106 THE LUNG AS A DRIVER OF RA-ASSOCIATED AUTOIMMUNITY}

\section{A. Catrina. Department of Medicine, Rheumatology Unit, Karolinska University} Hospital and Institutet, Stockholm, Sweden

Rheumatoid arthritis (RA) is a chronic inflammatory disease resulting from the complex interaction between genes and environment. In a large majority of patients this interaction leads to formation of disease-specific anti-citrullinated proteins antibodies (ACPA). Systemic autoimmunity may be triggered at mucosal sites (such as the lungs) long before the first signs of inflammation are starting in the joints. According to this model, smoking (and others environmental triggers) induces subclinical inflammation in the lungs, leading to increased local citrullination and formation of ACPA in genetically susceptible individuals. Lung changes on high-resolution computer tomography are present in both early-untreated ACPA positive RA and ACPA positive individuals at risk for but not yet having disease. Further, signs of subclinical inflammation and immune activation with germinal centers formation and ACPA enrichment is present in early untreated RA. Shared citrullinated targets have been described in the lungs and joints of patients with RA and more recent data unravels novel mechanisms showing how this extra-articular triggered autoimmunity progresses to joint-specific inflammation. Beside an initiating role, the lung might also be a secondary target for antibodies, especially in longstanding seropositive RA.

Disclosure of Interest: None declared

DOI: 10.1136/annrheumdis-2017-eular.7293 